\title{
Postnatal home visits from healthcare professionals show promise for preventing postnatal depression
}

Dennis CL. Psychosocial and psychological interventions for prevention of postnatal depression: systematic review. BMJ 2005;331:1-8.

\section{Do psychosocial and psychological interventions prevent postnatal depression?}

\section{METHODS}

Design: Systematic review.

Data sources: MEDLINE (1966-2004); EMBASE (1980-2004); CINAHL (1982-2004), Cochrane central register of controlled trials; Cochrane pregnancy and childbirth group trials register; Cochrane depression, anxiety, and neurosis trials register; hand searches of references lists and review articles; and consultation with experts in the field.

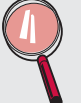

Study selection and analysis: Inclusion criteria: high quality (using Cochrane recommendations for assessment) randomised controlled trials (RCTs) of ante or early postnatal psychosocial or psychological interventions versus normal care for the prevention of postnatal depression. Interventions included antenatal and postnatal classes, lay home visits, early postpartum follow up, continuity of care, home visits, or interpersonal psychotherapy and debriefing. Exclusion criteria: purely educational interventions; RCTs in women with depression or depressive symptoms. Analysis: the pooled relative risk of postnatal depression was calculated using fixed and random effects models. Weighted mean differences were calculated where continuous measures were available. Heterogeneity was assessed using the $\mathrm{I}^{2}$ statistic.

国皿

Outcomes: Postnatal depression.

\section{MAIN RESULTS}

Fifteen RCTs ( $\mathrm{n}=7697$ women) met inclusion criteria. Overall, there was no significant effect of preventative interventions on the risk of postnatal depression ( 15 RCTs, RR $0.81,95 \%$ CI 0.65 to 1.02). At best, interventions achieved a short term reduction in postnatal depression, but this was no longer apparent at assessments beyond 16 weeks (at 0-8 weeks: eight RCTs, RR $0.65,95 \%$ CI 0.43 to 1.00 at 16+ weeks: seven RCTs, RR 1.02, 95\% CI 0.87 to 1.19). Analysis by type of intervention found that only home visits by a health professionals reduced postnatal depression (two RCTs; RR 0.68 , $95 \%$ CI 0.55 to 0.84 ). Individual interventions tended towards a risk reduction, but group interventions did not (individual, 11 RCTs: RR $0.76,95 \%$ CI 0.59 to 1.00; group, four RCTs: RR $1.03,95 \%$ CI 0.65 to 1.63). Interventions which started in the antenatal period and continued postnatally did not reduce postnatal depression (four RCTs, RR 1.21 , 95\% CI 0.93 to 1.59 ) while postnatal only interventions were effective ( 10 RCTs, RR 0.76 , 95\% CI 0.58 to 0.98 ). Interventions in women at "high risk" were more effective than those in unselected women from the general population ("high

For correspondence: Cindy-Lee Dennis, Assistant Professor, University of Toronto, Faculty of Nursing 50 St George Street, Toronto, ON, Canada M5S 3H4; cindylee.dennis@utoronto.ca

Sources of funding: none.

risk" women: seven RCTs, RR $0.67,95 \%$ CI 0.51 to 0.89 , general population: eight RCTs, RR $0.87,95 \%$ CI 0.66 to 1.16 )

\section{CONCLUSIONS}

There is no evidence to suggest that antenatal and postnatal classes, early postpartum follow up, continuity of care models, psychological debriefing in hospital, or interpersonal psychotherapy can prevent postnatal depression. There is some evidence that individual postnatal support from healthcare professionals for at risk mothers may be effective.

\section{NOTES}

The definition of postnatal depression varied across RCTs; most (10/ 15 RCTs) defined postnatal depression as a score of $>12$ on the Edinburgh postnatal depression scale.

\section{Commentary} he issue of psychosocial and psychological interventions is of interest and importance to sufferers and their families, clinicians and those that fund healthcare services. The review presents the rationale behind attempts to prevent postnatal depression in a clear and compelling way. There is consensus that postnatal depression is relatively common (around 13\%) and that it is associated with poor maternal and infant outcomes. Many psychosocial and psychological risk factors for postnatal depression have been identified. In addition to those noted by the author, depressive symptoms during pregnancy have been repeatedly shown to predict postnatal depressive symptoms and/or depressive diagnoses. ${ }^{1}$

Although a large number of potential studies were identified by the present study $(n=155)$, only 15 studies were suitable and sufficiently rigorous for inclusion in analyses. When interpreting the results of subgroup analyses (for example, impact of different types of interventions), it is important to keep in mind that often few studies were available for inclusion in these analyses.

The paper reaches a somewhat sobering conclusion. Despite the fact that it seems feasible and sensible to use psychosocial and psychological interventions to reduce the risk of postnatal depression, the evidence to support these approaches does not seem to be there currently. Although the author sees some promise in the provision of intensive, professionally based postpartum support, more studies are required to confirm this hope. The results of this review, and another recent review, ${ }^{2}$ suggest merit in evaluating interventions that are provided in the postnatal period and that target high risk groups. As the author of the present paper notes, it is also important that future trials include an economic analysis of relative costs and benefits.

Frances A Carter PhD

Department of Psychological Medicine, Christchurch School of Medicine and Health Sciences, University of Otago, Christchurch, New Zealand

1 Beck CT. A meta-analysis of predictors of postpartum depression. Nurs Res 1996;45:297-303

2 Lumley J, Austin MP, Mitchell C. Intervening to reduce depression after birth: a systematic review of the randomized trials. Int J Technol Assess Health Care 2004:20:128-44. 